\title{
Thick electrodes for Li-ion batteries: A model based analysis
}

\author{
Timo Danner ${ }^{\mathrm{a}, \mathrm{b}, *}$, Madhav Singh ${ }^{\mathrm{c}}$, Simon Hein ${ }^{\mathrm{a}, \mathrm{b}}$, Jörg Kaiser ${ }^{\mathrm{c}}$, Horst Hahn ${ }^{\mathrm{b}, \mathrm{c}}$, Arnulf Latz ${ }^{\mathrm{a}, \mathrm{b}, \mathrm{d}}$ \\ ${ }^{a}$ Institute of Engineering Thermodynamics, German Aerospace Center (DLR), Pfaffenwaldring 38-40, 70569 Stuttgart, Germany \\ ${ }^{b}$ Helmholtz Institute Ulm for Electrochemical Energy Storage (HIU), Helmholtzstraße 11, 89081 Ulm, Germany \\ ${ }^{c}$ Karlsruhe Institute of Technology (KIT), Institute of Nanotechnology, Hermann-von-Helmholtz Platz 1, 76344 Eggenstein-Leopoldshafen, \\ Germany \\ ${ }^{d}$ Institute of Electrochemistry, University of Ulm, Albert-Einstein-Allee 47, 89081 Ulm, Germany
}

\begin{abstract}
Li-ion batteries are commonly used in portable electronic devices due to their outstanding energy and power density. A remaining issue which hinders the breakthrough e.g. in the automotive sector is the high production cost. For low power applications, such as stationary storage, batteries with electrodes thicker than $300 \mu \mathrm{m}$ were suggested. High energy densities can be attained with only a few electrode layers which reduces production time and cost. However, mass and charge transport limitations can be severe at already small C-rates due to long transport pathways. In this article we use a detailed 3D micro-structure resolved model to investigate limiting factors for battery performance. The model is parametrized with data from the literature and dedicated experiments and shows good qualitative agreement with experimental discharge curves of thick NMC-graphite Li-ion batteries. The model is used to assess the effect of inhomogeneities in carbon black distribution and gives answers to the possible occurrence of lithium plating during battery charge. Based on our simulations we can predict optimal operation strategies and improved design concepts for future Li-ion batteries employing thick electrodes.
\end{abstract}

Keywords: Li-ion batteries, continuum modeling, micro-structure resolved simulation, thick electrodes

\section{Introduction}

The energy density of Li-ion cells, one of if not the most decisive parameter, is constantly being improved by a number of measures. On the one hand, active anode and cathode materials are tuned to provide higher cell voltages and higher charge densities. On the other hand, cell manufacturers minimize the fraction of inactive components including reduction of binder and conducting aids in the electrode layers, reduction of pore volume in highly calendered electrodes, use of thin separators and anode to cathode balancing factors close to unity. Furthermore, thick electrode layers have a favorable electrode to current collector ratio per stack volume and additionally provide reduced cell manufacturing costs due to fewer cutting and stapling steps.

In this context we have recently presented a study where we compared $70 \mu \mathrm{m}$ and $320 \mu \mathrm{m}$ thick graphite and NMC(111) electrode layers [1]. While it was shown that the energy density can be improved, it also appeared that electrokinetics of thick electrodes suffer from limitations in electron and mass transport which becomes critical during charging when conditions evolve under which lithium plating can occur in the regions of the anode close to the separator even at moderate $\mathrm{C}$ rates. This was presumably the reason for the short cycle life observed with the cells consisting of thick anodes and cathodes.

In order to understand these effects in detail, different approaches are generally being pursued. The straight forward one commonly used but resource intensive is the measurement of test matrices set up from parameters such as temperatures, C-rate, depth of discharge and others with one or more cells per matrix element accompanied by anteand postmortem analysis. This works well when a sufficient amount of comparable cells, preferably commercially

\footnotetext{
${ }^{*}$ Corresponding author:

Email address: Timo.Danner@DLR.de (Timo Danner)

URL: http://www.dlr.de/tt/en/ (Timo Danner)
} 
produced cells are available. Another approach is to directly look into the battery during operation. In the literature several in-operando techniques were suggested to study specific aspects of Li-ion batteries. However, often modifications of the cell design are necessary in order to fit the sample to the needs of the measurement device, such as restrictions in sample size. This complicates a direct transfer of results between different cell designs.

Detailed theoretical studies provide an interesting alternative and a variety of models were suggested in the literature for Li-ion batteries. They range from simple and efficient equivalent circuit and performance models [2, 3] to physically motivated electrochemical [4, -8] and thermal [9, 10] models. Important aspects affecting the performance and lifetime of the cell, such as dendrite formation [11, 12], SEI growth [13]-15], lithium plating [16, 17], mechanical stress and crack formation [18-20], and many more were studied in detail and help to improve our understanding of relevant process for a successful operation of Li-ion batteries. In this article we present detailed 3D micro-structure resolved simulations of half-cells as well as full battery cells consisting of thick NMC cathodes and graphite anodes. In our framework for numerical simulations BEST we are able to distinguish between active material, binder, and conductive additive such as carbon black which allows us to study the effect of local conditions and inhomogeneities on global cell performance. A focus of the simulations presented in this article is set on the investigation of the influence of an inhomogeneous carbon black distribution. Carbon black consists of particles of high dispersion with a strong tendency to form stable agglomerates. In order to function as an electronic conducting aid in Li-ion battery electrodes, these agglomerates must be cracked during the electrode slurry preparation step by the usage of mechanical stirring force and then be coated on the active material particles so that they provide interconnected pathways of high electron conductivity from the substrate foil to ideally every single active material particle [21]. Thus the destruction of agglomerates and the coating are sensitive steps and small deviations from the optimal process parameters could, among others, negatively affect local potential distributions in the electrode layers and lead to performance losses particularly in the case of thick electrode layers. Moreover, we study the occurrence of lithium plating during battery charge which is regarded as a major degradation mechanism. The basis for qualitative predictions on a complicated system such as a Li-ion battery is a quantitative parameterization of the model. Most of the parameters are either taken from the literature or our own dedicated experiments. For the estimation of kinetic parameters we present an approach based on supplemental simulations with a reduced and computationally efficient $1+1 \mathrm{D}$ model. The article is structured as follows:

First, we present the setup and results of half- and full cell measurements on NMC/graphite cells which provide the basis for a sound parameterization and validation of our model (Section 2). The model layout and governing equations are summarized in Section 3 . Finally, we present in Section 4 results of our simulations and discuss in detail the effect of local inhomogeneities in carbon black distribution and operating conditions on the performance of the battery.

\section{Experiments}

Graphite and NMC(111) were used to prepare $70 \mu \mathrm{m}$ and $320 \mu \mathrm{m}$ thick anode and cathode layers with high active material contents. They were tested in both half cell and full cell configuration. The electrochemical characterization and all further details regarding the experimental setup and cycling protocols can be found in [1]. Representative charge and discharge curves are shown in the course of model parameterization and validation. The open circuit voltages in Figure 1 were recorded in half cell configuration on $70 \mu \mathrm{m}$ thick electrodes vs. lithium metal. After three charge and discharge cycles the cells were charged stepwise by $2.5 \%$ SOC followed by a 30 min resting period after which the cell voltage was recorded. After fully charging the procedure was repeated in discharge direction to account for a possible hysteresis. The data sets for lithiation and delithiation are fitted with an empirical correlation given by

$$
\begin{aligned}
\mathrm{OCV}_{\mathrm{NMC}}(\mathrm{soc}) & =7.9760-5.5419 \cdot \mathrm{soc}+5.2824 \cdot \operatorname{soc}^{1.0700} \\
& -1.0556 \cdot 10^{-04} \cdot \exp (124.7407 \cdot \operatorname{soc}-114.2593) \\
& -4.0446 \cdot \operatorname{soc}^{0.0766} .
\end{aligned}
$$

In order to extend the range of the correlation the data was extrapolated to voltages above the upper cut-off limit of 4.2 $\mathrm{V}$ of the half-cell measurements. Figure 1 demonstrates that the data can be well described by Eq. (1). At SOC=0 and $\mathrm{SOC}=1$ we enforce a strongly diverging $\mathrm{OCV}$ curve to improve the stability of the 3D simulations if a particle is fully de-/lithiated. From these OCV measurements we also deduce a maximum amount of lithium $c_{\mathrm{s}}^{\max }=36224$ mol 


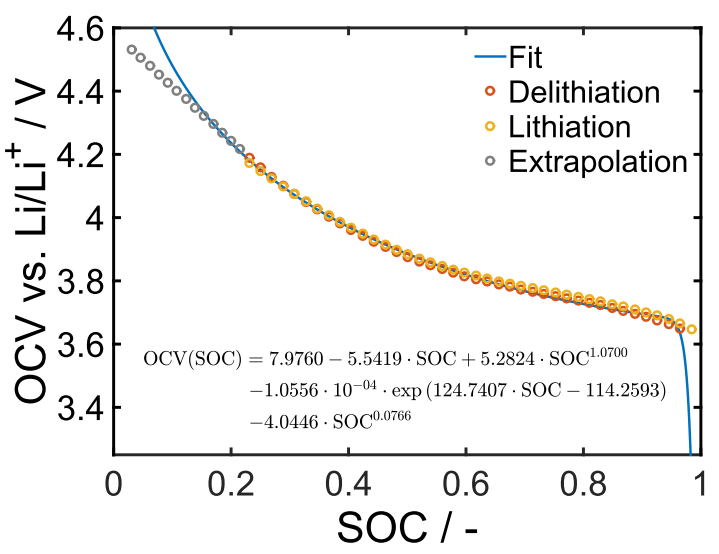

Figure 1: OCV curves determined by pulsed lithiation (yellow) and delithiation (red) measurements. Gray symbols represent extrapolated data points beyond the cut-off voltage of $4.2 \mathrm{~V}$. The blue line shows a fit to the electrochemical data as given by Eq. 1 .

$\mathrm{m}^{-3}$ which can be stored in the active material in the given potential window.

In a second experiment we determine the effective conductivity of dry NMC electrodes. Disc shaped samples with a diameter of $11 \mathrm{~mm}$ were clamped between two nickel electrodes before the AC impedance at $5 \mathrm{kHz}$ was measured (Gamry potentiostat interface 1000). Varying specific conductivities of 0.6 and $1.89 \mathrm{~S} \mathrm{~m}^{-1}$ were found for the $70 \mu \mathrm{m}$ and $320 \mu \mathrm{m}$ thick electrode, respectively. An explanation for this deviation might be a strong contribution of a contact resistance or an inhomogeneous carbon black and binder distribution in the thick electrodes.

\section{Simulation methodology}

In this section we give details on our approach for the simulation of thick NMC-graphite Li-ion batteries. First, we explain our method of structure generation and characterization. The resulting geometry and effective transport parameters are input to our electrochemical 1+1D and 3D simulations. 1+1D simulations are mainly used for an efficient determination of kinetic parameters which are needed in our detailed 3D micro-structure resolved simulations.

\subsection{Structure generation and characterization}

Realizations of electrode micro-structures are an important corner stone for predictive micro-structure resolved simulations [22, 23]. In this study we employ synchrotron radiation X-ray tomographies of NMC battery electrodes provided open source by Ebner et al. [22]. The data matches favorably with our electrodes regarding composition, porosity, and particle size distribution. The reconstructions with dimensions of $\sim 700 \times 700 \times 70 \mu \mathrm{m}$ are imported in the software GeoDict [24] for a first structural characterization of transport parameters and specific surface area. The results show that the structure is rather isotropic which allows us to rotate the structure by $90^{\circ}$ in order to generate a realization of a thick electrode. An additional coarsening step is needed to reduce the computational load of the 3D simulations and the final voxel length or resolution of our simulations is $1.48 \mu \mathrm{m}$. The binder phase is not resolved in the tomography images. However, it is known that the binder distribution has an influence on the performance of the cell since it provides mechanical stability but blocks active surfaces. In our approach we distribute the binder in its respective weight percentage at positions in the electrode where the distance between neighboring active particles is small. One of the challenges in micro-structural simulations is a mismatch in the scales of the different materials, such as active particles $\mathrm{d}_{50} \approx 11 \mu \mathrm{m}$ and conductive additive $\mathrm{d}_{50} \approx 100 \mathrm{~nm}$. A resolution of the carbon black particles is not feasible due to the high computational load and a lack of reliable image data. In our model we assume a continuous surface layer with high electronic conductivity instead of resolving individual particles.

The negative electrode consists of artificial graphite with an average particle diameter of $24 \mu \mathrm{m}$. Unfortunately, there was no tomography data available for our structural model. Therefore, we use the software GeoDict to construct 
an electrode realization based on the data sheets provided by material suppliers and SEM images [1]. The virtual model electrode consists of convex polyhedrons with a defined distribution of particle size and shape in order to get a realistic representation of the electrode geometry. In a second step the binder phase is distributed in the same manner as described for the positive electrode. Deviations in the transport behavior compared to the real electrode can be expected and tomographies of graphite electrodes are an important direction for future research. In Section 4.1 we investigate the sensitivity of our simulations towards effective transport parameters in order to estimate the error of our simulations.

In the full cell simulations the area and thickness of the graphite anode are increased in order to avoid edge effects and to take into account the balancing factor of the electrodes. At the cathode a thin layer of insulating material surrounding the electrode structure in $y$ and $z$ direction is assumed which is meant to represent the cell housing. Images of NMC and graphite micro-structures are provided at the top of Figure 2 and transport as well as structural parameters are summarized in Table 1 .

\section{2. $1+1 D$ model}

The modeling of Li-ion batteries was pioneered by Newman and co-workers [25, 26] and their work provides the basis for a variety of continuum models suggested in the literature [27, 9, 5]. This pseudo-2D model is state of the art and a derivation of governing equations with the help of volume averaging techniques is given in [28] and [10]. A detailed description of constitutive equations is not in the focus of this work, however, we provide a brief overview of the most important model features and summarize the equations in Table 2

In the pseudo-2D (1+1D) approach the transport of lithium ions by diffusion and migration in the electrolyte is described by a volume-averaged material balance as given by Eq. (6). The potential of the electrolyte which is needed to describe the migrative flux is obtained by a charge balance under the assumption of electro-neutrality (cf. Eq. (8)). At the cathode and anode current collector we assume no-flux boundary conditions $\left(\partial c_{\mathrm{e}} / \partial x=\partial \phi_{\mathrm{e}} / \partial x=0\right)$. In each control volume a representative active particle is modeled to take into account the diffusion of lithium in the active material (Eq. (14)). The de-/intercalation of lithium ions on the surface of the active particles

$$
\mathrm{Li}^{+}+\mathrm{e}^{-} \rightleftharpoons \mathrm{Li}
$$

is then typically described by the Butler-Volmer equation

$$
\begin{aligned}
i_{\mathrm{se}} & =i_{0}\left[\exp \left(\frac{\alpha F}{R T} \eta\right)-\exp \left(-\frac{(1-\alpha) F}{R T} \eta\right)\right] \\
& =i_{00} c_{\mathrm{e}}^{\alpha} c_{\mathrm{s}}^{\alpha}\left(c_{\mathrm{s}}^{\max }-c_{\mathrm{s}}\right)^{1-\alpha}\left[\exp \left(\frac{\alpha F}{R T} \eta\right)-\exp \left(-\frac{(1-\alpha) F}{R T} \eta\right)\right] .
\end{aligned}
$$

The associated flux of lithium $i_{\mathrm{se}} / \mathrm{F}$ is used as boundary condition for Eq. (14) at the particle surface. At the particle center no-flux boundary conditions apply due to symmetry reasons $\left(\partial c_{\mathrm{s}} / \partial r=0\right)$. The resulting flux of lithium ions and electrons enter as a source term in the material balance of lithium ions (Eq. (6)) and the charge balance of the solid phase (Eq. (16), respectively. In the half-cell setup the negative electrode consists of a lithium metal foil. We model the lithium electrode as an infinite reservoir of lithium which is stripped and deposited with a constant exchange current density $i_{0}=i_{00}=10 \mathrm{~A} \mathrm{~m}^{-2}[29]$.

The most prominent advantage of this type of model is its computational efficiency. Full charge/discharge simulations are computed in only a few seconds to minutes on a standard desktop computer. Therefore, we employ the $1+1 \mathrm{D}$ model in our work for the efficient estimation of model parameters by a fit to experimental data. The governing equations and a non-linear least squares algorithm for parameter estimation are implemented in Matlab.

\section{3. $3 D$ model}

For the 3D micro-structure resolved simulations we use our software BEST (Battery and Electrochemistry Simulation Tool) which is developed in a joint effort by DLR/HIU and Fraunhofer ITWM Kaiserslautern [30]. BEST is a finite volume implementation based on the CoRheos framework for complex and granular flow developed at Fraunhofer. The governing equations are derived in a rigorous approach from fundamental non-equilibrium thermodynamics. A detailed derivation which also includes the description of a thermal model can be found in [8, 10]. In this article we 
focus on isothermal simulations and the relevant equations are summarized in Table 2.

The model consists of a similar set of governing equations as introduced in the previous paragraph describing the transport of mass and charge in the electrolyte (Eq. (7) and Eq. (9)) and solid material (Eq. (15) and Eq. (17)). However, there are some fundamental differences between volume-averaged and micro-structure resolved models which will be explained in detail in the following paragraph. Eq. (7) describes the temporal evolution of lithium ion concentration in the liquid electrolyte

$$
\frac{\partial c_{\mathrm{e}}}{\partial t}=-\nabla \cdot\left(-D_{\mathrm{e}} \nabla c_{\mathrm{e}}+\frac{t_{\mathrm{Li}} i_{\mathrm{e}}}{F}\right)
$$

Compared to Eq. (6) this equation does not contain a source term related to the de-/intercalation of lithium. In a homogenized model, like the 1+1D model described above, solid and liquid phases coexist in each voxel with their respective volume fraction $\varepsilon_{i}$. The volume averaging transforms the interfacial flux into a volume source term weighted with a volume specific surface area $a^{v}$. In micro-structure resolved simulations each voxel can be clearly assigned to a certain phase or material with defined interfaces between neighboring voxels. Therefore, the de-/intercalation current at the electrode-electrolyte interface is modeled as an interface flux [8] given by

$$
n \cdot \frac{i_{\mathrm{e}}}{F}=n \cdot \frac{i_{\mathrm{s}}}{F}=\frac{i_{\mathrm{se}}}{F},
$$

where the area normal $n$ points from the solid to the electrolyte phase and $i_{\text {se }}$ is calculated from the Butler-Volmer expression in Eq. (3). The same applies for the charge balance in the electrolyte and solid phase. Moreover, since each voxel can be assigned to a certain material or phase the transport properties in the bulk of the respective material are used in Eqs. (7), (9), (15), and (17). In the 1+1D model effective transport parameters are needed as a result of the volume averaging approach. Details on the underlying theory and simulation strategies can be found in [8, 10, 17]. The simulations are performed directly on the electrode structures presented in Section 3.1. BEST is able to handle multiple solid phases, such as binder, carbon black, and active material. For each material transport parameters $\left(D_{\mathrm{s}}\right.$, $\kappa)$ need to be defined. The insulating nature of the binder phase is described by no-flux conditions to neighboring phases, whereas the surface layer of carbon black (cf. Section 3.1) is modeled as active material with high electric conductivity. In this case the transport of lithium and electrons is assumed to be continuous at phase boundaries and the charge transfer reaction between the electrolyte phase and active material is described by the Butler-Volmer equation (Eq. (3)). The micro-structure of the separator is not resolved explicitly in this study and we use effective transport parameters for the electrolyte phase in this region. At the boundary of the through plane direction which is denoted by $x$ in the 1+1D case we model current collectors with high electric conductivity and enforce no-flux conditions for lithium in the solid phase and lithium ions in the electrolyte. In the directions orthogonal to $x$ we also enforce no-flux conditions at the boundary of the computational domain since the electrode reconstructions are not periodic in $y$ and $z$. Test simulations using periodic boundary conditions showed a negligible influence on simulation results even for inhomogeneous carbon black distribution.

3D simulations are performed in parallel on 4 processors on the Justus cluster hosted by the Ulm University. The computation time is in the order of 1 day for full-cell simulations of thick Li-ion batteries. Results of our simulations are presented in Section 4

\section{Results and discussion}

In this section we present results of our measurements and simulations of thick Li-ion batteries. First, we describe in detail our parameterization methodology and validate the model against experimental data of half- and full-cell measurements. The validated 3D model is used for a detailed analysis of transport processes within the electrodes of thick Li-ion batteries. In this context we set a focus on the influence of an inhomogeneous carbon black distribution on battery performance and the possible degradation of battery capacity and lifetime due to lithium plating.

\subsection{Model parameterization and validation}

A reliable parameterization is an important step for predictive simulations. Except for one all of the parameters of our models are either obtained from the literature or dedicated measurements. In order to ensure a consistent parameterization of the $1+1 \mathrm{D}$ and $3 \mathrm{D}$ model we determine effective transport parameters for the structures presented in 


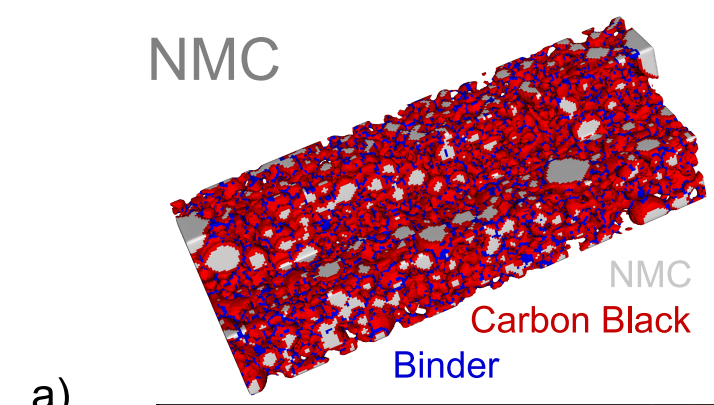

a)

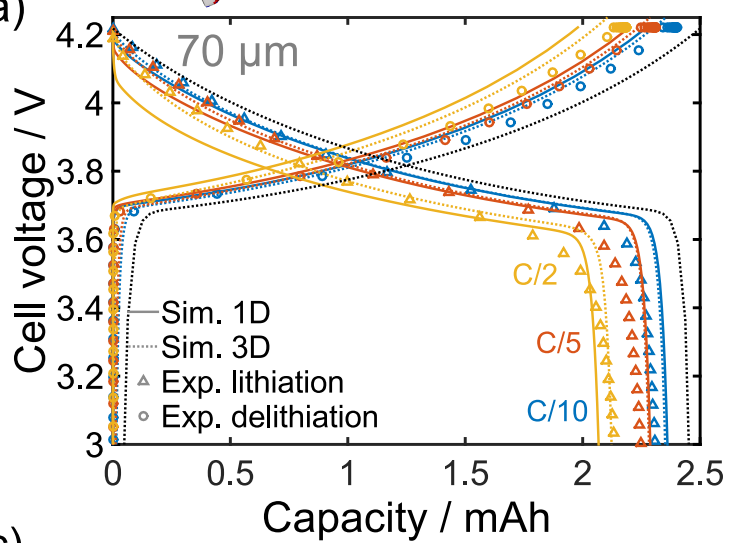

c)

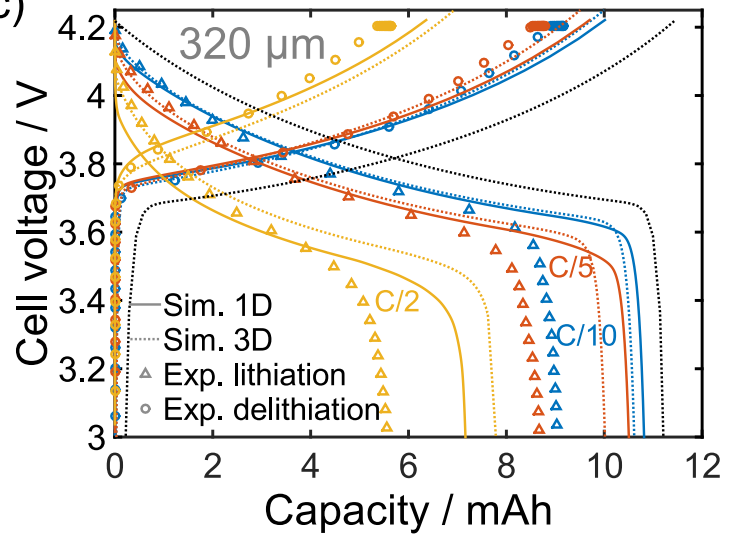

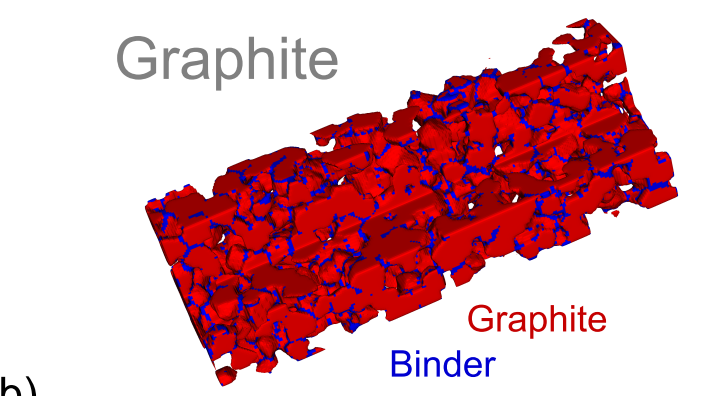

b)

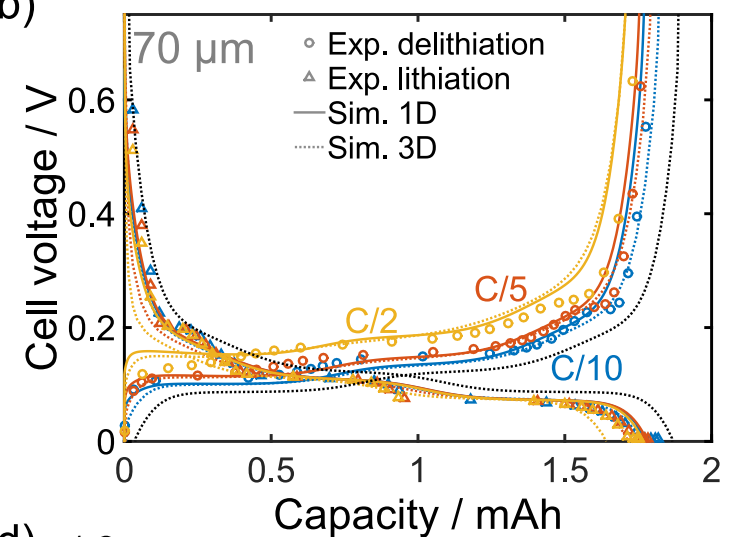

d)

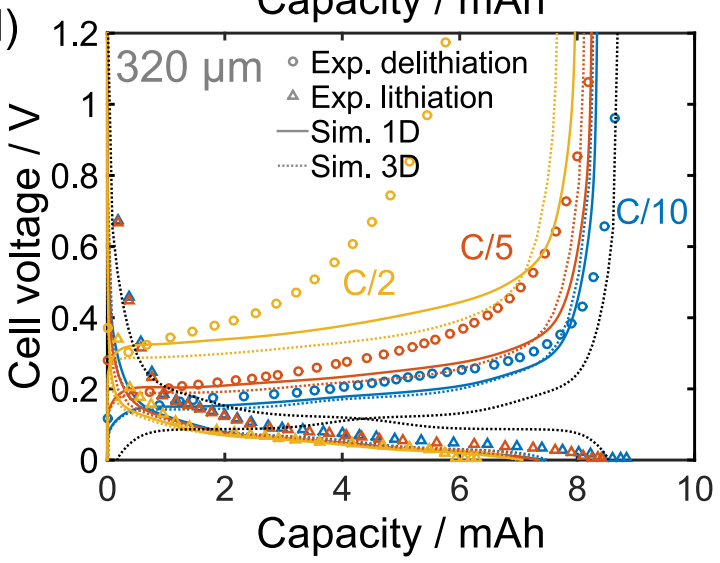

Figure 2: Simulation results of electrodes with a thickness of $70 \mu \mathrm{m}$ (top) and $320 \mu \mathrm{m}$ (bottom) in half-cell configuration. The dotted line gives the corresponding OCV curves as reference. The micro-structures of the NMC (left) and Graphite (right) electrode structures which are used in 3D simulations and for the determination of effective transport parameters are shown at the top the Figure.

Section 3.1 in GeoDict. The negative electrode consisting of graphite $\left(100 \mathrm{~S} \mathrm{~m}^{-1}[31]\right)$ and insulating binder has an effective conductivity $\sigma^{\text {eff }} \approx 10.1 \mathrm{~S} \mathrm{~m}^{-1}$. At the positive electrode we assume $\sigma^{\text {eff }} \approx 1 \mathrm{~S} \mathrm{~m}^{-1}$ which is in between the experimental data points for the thin and thick electrode (cf. Section 2). The conductivity of the carbon black surface layer $\left(17.1 \mathrm{~S} \mathrm{~m}^{-1}\right)$ is adjusted to match the electrode conductivity which is determined by the bulk conductivities of NMC (0.023 S m$\left.~^{-1}[32]\right)$, carbon (100 S m$~_{-1}^{[31]}$ ), and insulating binder. Bulk properties of the liquid electrolyte, such as conductivity, $\mathrm{Li}^{+}$diffusion coefficient, and transference number are reported in Ref. [33]. The OCV curves of graphite are well studied in the literature and we use a correlation proposed by Safari et al. [27]. The corresponding exchange current density and lithium diffusion coefficient are taken from a parameter study by Vazquez-Arenas et al. [34] on NMC/graphite cells, which give a good agreement to our half-cell measurements (see Figure 2 b)). However, their parameterization of the NMC electrode gives unsatisfactory results. Therefore, we use the OCV curves presented in Section 2 in our simulations and determine the exchange current density of NMC $i_{00}=5.06 \cdot 10^{-6} \mathrm{~m}^{2.5} \mathrm{~mol}^{-0.5} \mathrm{~s}^{-1}$ 
a)

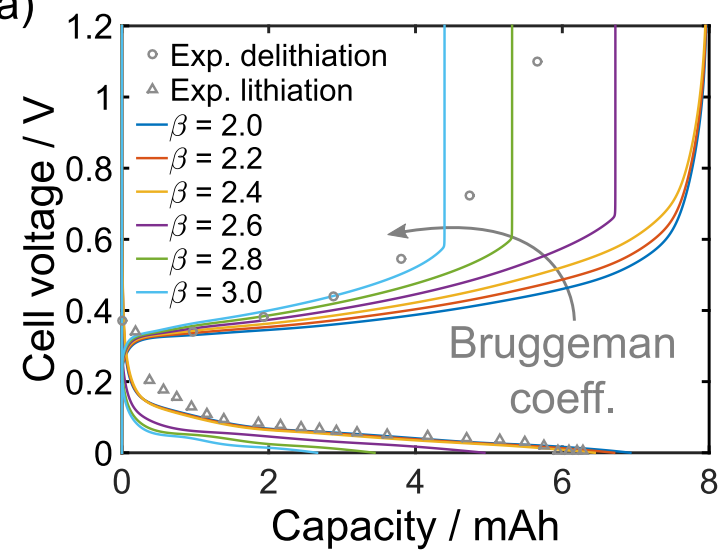

b)

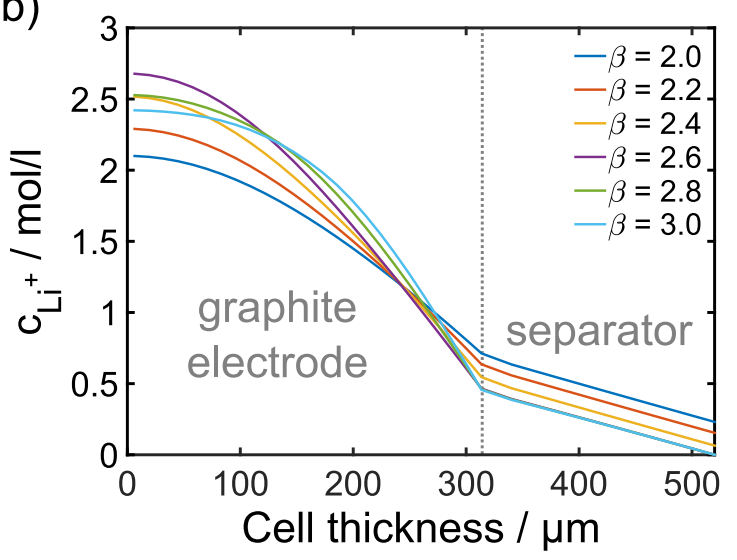

Figure 3: Results of $1+1 \mathrm{D}$ delithiation simulations of the $320 \mu \mathrm{m}$ graphite electrode at a rate of $\mathrm{C} / 2$ for increasing Bruggeman coefficient corresponding to an increase in transport resistance.

by a fit of the 1+1D model to experimental data of half-cell measurements on a $70 \mu \mathrm{m}$ thick electrode (cf. Figure $2 \mathrm{a}$ )). The diffusion coefficient of lithium is parametrized by simulating the stepwise charge and relaxation protocol which was used in the measurement of OCV curves. The estimated diffusion coefficient $D_{\mathrm{s}} \approx 2 \cdot 10^{-15} \mathrm{~m}^{2} \mathrm{~s}^{-1}$ matches with the value found by Dees et al. [35] for the simulation of electrochemical impedance spectra. All model parameters and corresponding sources are summarized in Table 3

Figures 2 a)-d) show de-/lithiation curves of NMC (left) and graphite (right) half-cell measurements on electrodes with 70 and $320 \mu \mathrm{m}$ thickness. The measurements are performed with increasing $\mathrm{C}$-rate $(\mathrm{C} / 10, \mathrm{C} / 5, \mathrm{C} / 2,5$ cycles each) in discharge direction or delithiation and lithiation direction of the graphite and NMC half-cell, respectively. The $\mathrm{C}$-rate of the charge process is kept constant at $\mathrm{C} / 10$. NMC half-cells and full-cell measurements are recorded galvanostatically with a lower and upper cut-off voltage of 3 and $4.2 \mathrm{~V}$, respectively. The graphite half-cells are operated in a potential window between $5 \mathrm{mV}$ and $2.0 \mathrm{~V}$. In general, we can report favorable agreement between measurements and simulations, especially for thin electrodes (Figure 2 a) and b)). Differences between 1+1D and 3D simulations are minor and become visible at high current densities only. This result shows that efficient 1+1D simulations can be used to obtain a first estimate of parameters for the detailed 3D model. However, at high current densities local effects which are not resolved in the 1+1D model become more prominent and the deviation to the micro-structure resolved simulations increases. This effect was also found by Goldin et al. [7] in their study on model electrodes consisting of spherical particles. Figures 2 c) and d) show de-/lithiation curves of thick electrodes which demonstrate a good qualitative agreement between measurements and simulations. In the case of NMC (Figure 2 2 )) the predicted capacity is higher compared to the experimental data. Deviations to the optimal electrode structure due to the challenging production process of thick electrodes might be responsible for this mismatch. This could cause fluctuations in electrode loading or an inhomogeneous binder as well as carbon black distribution. The first case can be almost excluded since our measurements showed a good reproducibility. An inhomogeneous binder distribution might block active surfaces and transport pathways for electrons and lithium ions. An inhomogeneous carbon black distribution might lead to insufficiently connected areas of the electrode. Both cases will effectively lead to a loss in cyclable active material and, therefore, capacity. The effect of inhomogeneous carbon black distribution will be discussed in detail in Section 4.3. It has to be noted, that a scaling of the simulated and measured voltage curves with the maximum theoretical and practical capacity, respectively, yields better agreement between simulation and experiment at all rates. An analysis of the 3D concentration distribution of lithium ions in the electrolyte reveals a massive transport limitation leading to vanishing lithium ion concentration in large parts of the NMC electrode close to the current collector. This causes a disproportionately high capacity loss at a lithiation rate of $\mathrm{C} / 2$ and is an indication that thick electrodes in the current design are not suitable for high power applications with $\mathrm{C}$-rates much larger than $\mathrm{C} / 5$.

A similar behavior is found for the delithiation of graphite at high C-rates as shown in Figure 2 d). However, the capacity loss which is found in the experiments is more severe than predicted by our simulations. A possible explanation 
a)

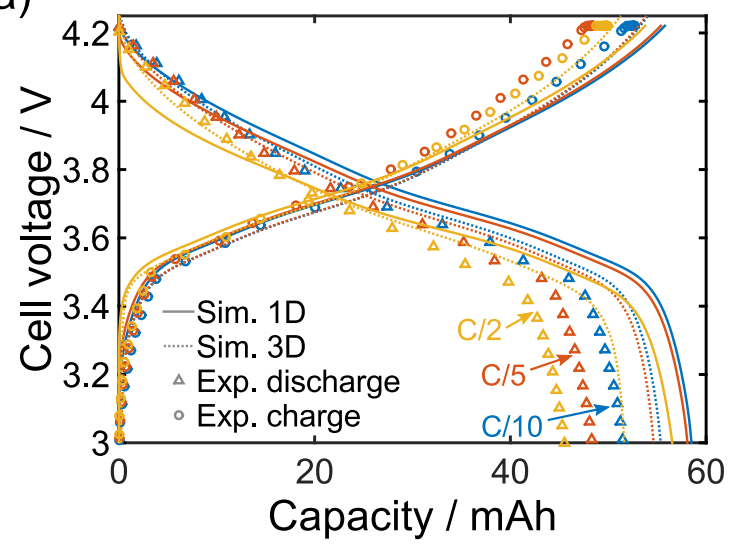

b)

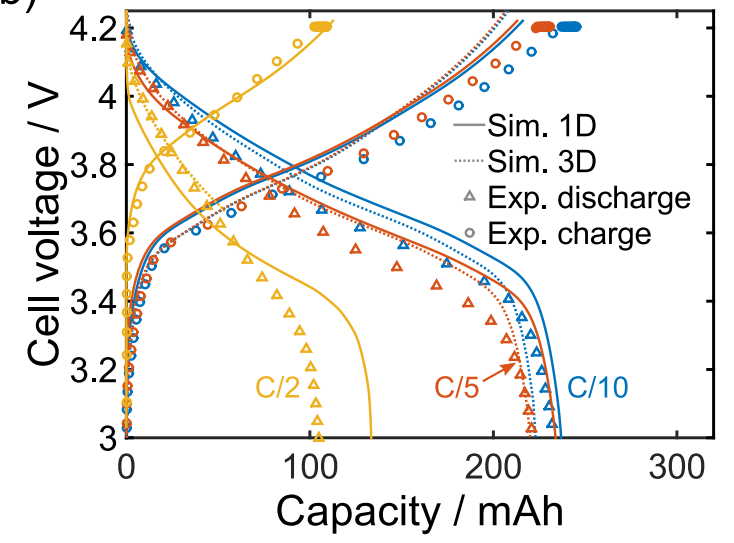

Figure 4: Simulation results of the full battery cell. Left: Thin electrodes Right: Thick electrodes.

might be that the transport resistance in the electrolyte is higher than expected. As discussed in Section 3.1 the anode micro-structure is reconstructed based on their macroscopic properties and SEM images. Local inhomogeneities and bottle-necks for lithium ion transport are not captured in this approach. In order to assess the effect of a higher transport resistance we vary the Bruggeman coefficient $\beta$ in $1+1 \mathrm{D}$ simulations. Figure 3 a) shows the resulting voltage curves at $\mathrm{C} / 2$ rate. The simulations show that transport limitations become prominent for $\beta \geq 2.6$ resulting in a high capacity loss. Figure $3 \mathrm{~b}$ ) gives the corresponding concentration profiles at the end of the lithiation process. The simulations predict that a high transport resistance will lead to a depletion of lithium in front of the lithium metal anode. This leads to a sharp increase in cell voltage due to our assumption of constant exchange current density as observed in Figure 3 a). Simulations with an exchange current density proportional to the concentration of lithium ions showed a smoother increase but qualitatively similar results. Values of $\beta \geq 2.6$ are rather high and further investigations on the micro-structure of graphite electrodes are needed to resolve this issue. Another possible source for the capacity loss could be the deposition of metallic lithium during the lithiation of graphite. In our simulations we do not find an indication for this degradation process at the relatively moderate lithiation current of a $\mathrm{C} / 10$ rate. A detailed analysis of lithium plating in the full battery cell is done in Section 4.4. Interestingly, we find in our simulations a large contribution of the charge transfer resistance at the lithium metal electrode to the overall cell resistance. Although lithium deposition kinetics are generally several orders of magnitude faster than intercalation kinetics the small active surface area limits the deposition rate. In the NMC electrode which has a lower porosity and higher tortuousity this effect was found to be negligible. In this case the charge transport resistance is larger relative to the charge transfer resistance at the lithium metal electrode.

\subsection{Full-cell simulations}

Figure 4 presents results of full battery cell simulations consisting of thin (left) and thick (right) electrodes. Especially, the simulation results of the thick cell are in good qualitative agreement with the experimental data. This indicates that our model captures most of the relevant effects determining the performance of the battery. At high discharge rates we find a severe transport limitation of lithium ions as it was discussed above for NMC half-cells. This effect imposes the use of very small time steps in the simulation and the calculations had to be stopped half-way during discharge. Modifications of the code and/or solution strategy will be necessary to overcome this limitation for future work. However, it has to be pointed out that also the operation of the 'real' battery cell under these extreme conditions should be avoided in practical applications.

The validated 3D model gives the opportunity to study the influence of local conditions and inhomogeneities on cell performance. These studies are not possible in standard 1+1D simulations and are a unique strength of micro-structure resolved simulations. In the remainder of this section the model will be used to analyze in detail the effect of inhomogeneities in carbon black distribution in the NMC electrode and the occurrence of lithium plating in the graphite electrode during battery charge. 


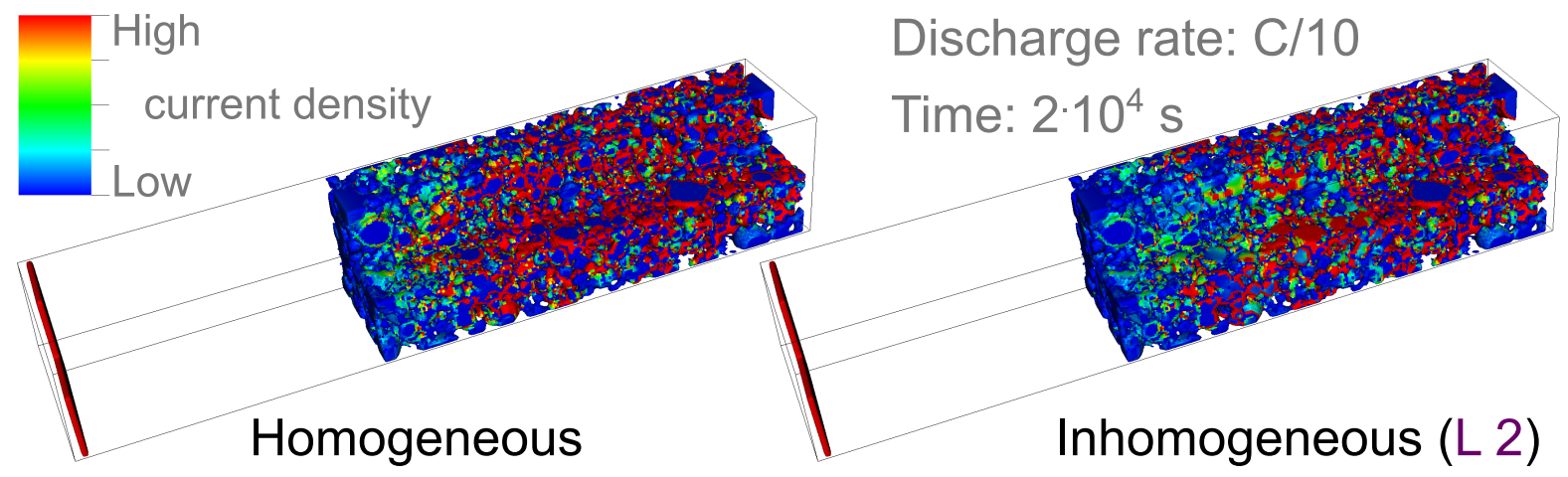

a)

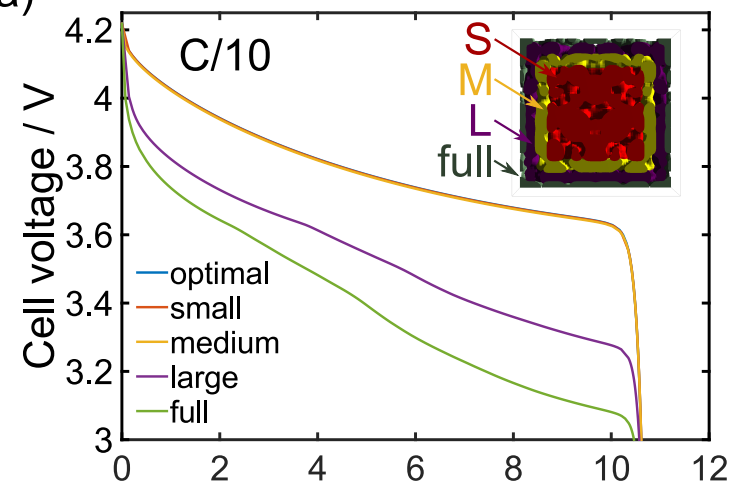

c)

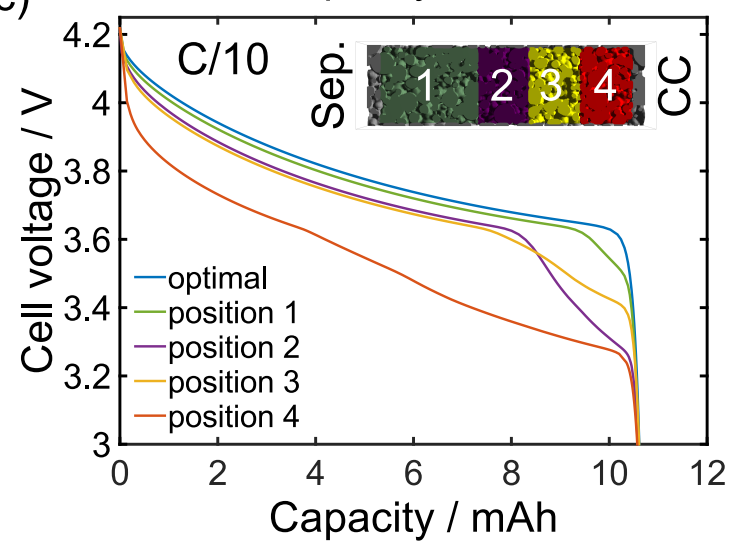

b)
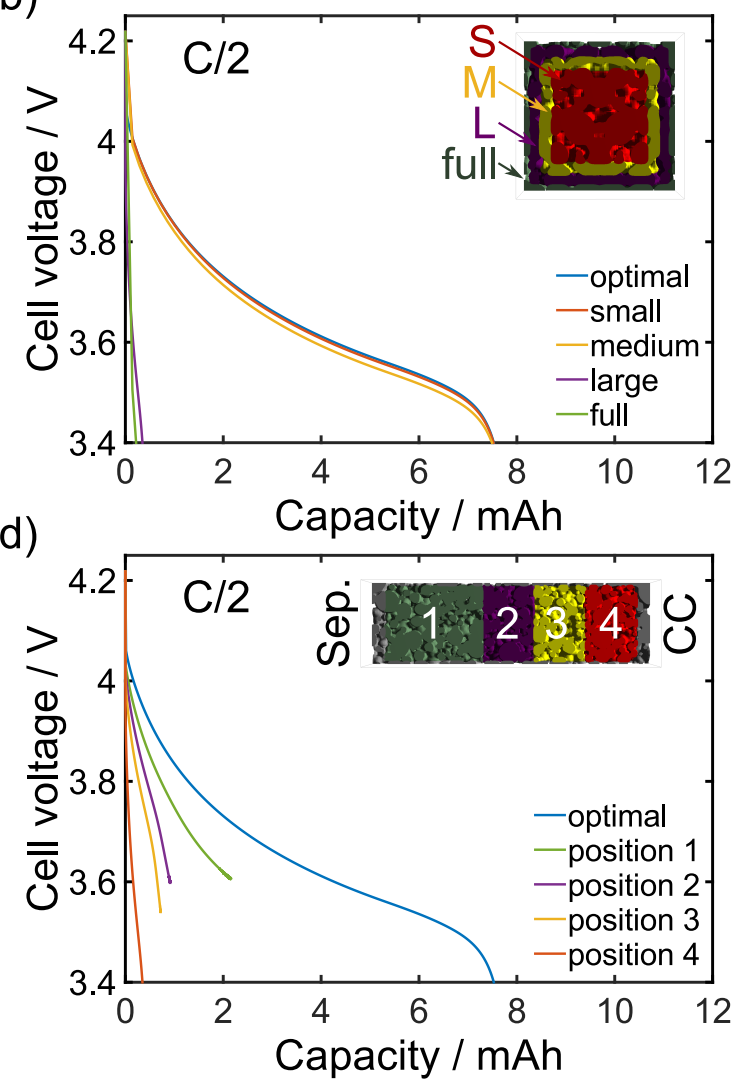

Figure 5: Lithiation curves of thick NMC electrodes in half-cell configuration. Graphs show the influence of an inhomogeneous soot distribution for varying defect sizes at position '4' (5 a) and b)) and a large defect at varying defect positions (5 c) and d)) relative to the cathode current collector (CC).

\subsection{Carbon black distribution}

The fabrication process of battery electrodes with high areal loading is very challenging and a scale-up of the electrode thickness is not straight forward. New production parameters or even new techniques will need to be developed for e.g. the drying process of thick electrodes. Inhomogeneities in binder or carbon black distribution can affect the life time and capacity of the battery. In this section we investigate the influence of carbon black distribution on the performance of the NMC electrode. In the optimal case we assume a perfect conductive layer on all NMC particles. As inhomogeneity we define particles which do not have a layer of conductive carbon black on the surface and the whole particle has the low conductivity of NMC. In our simulations we vary both the position and size of 


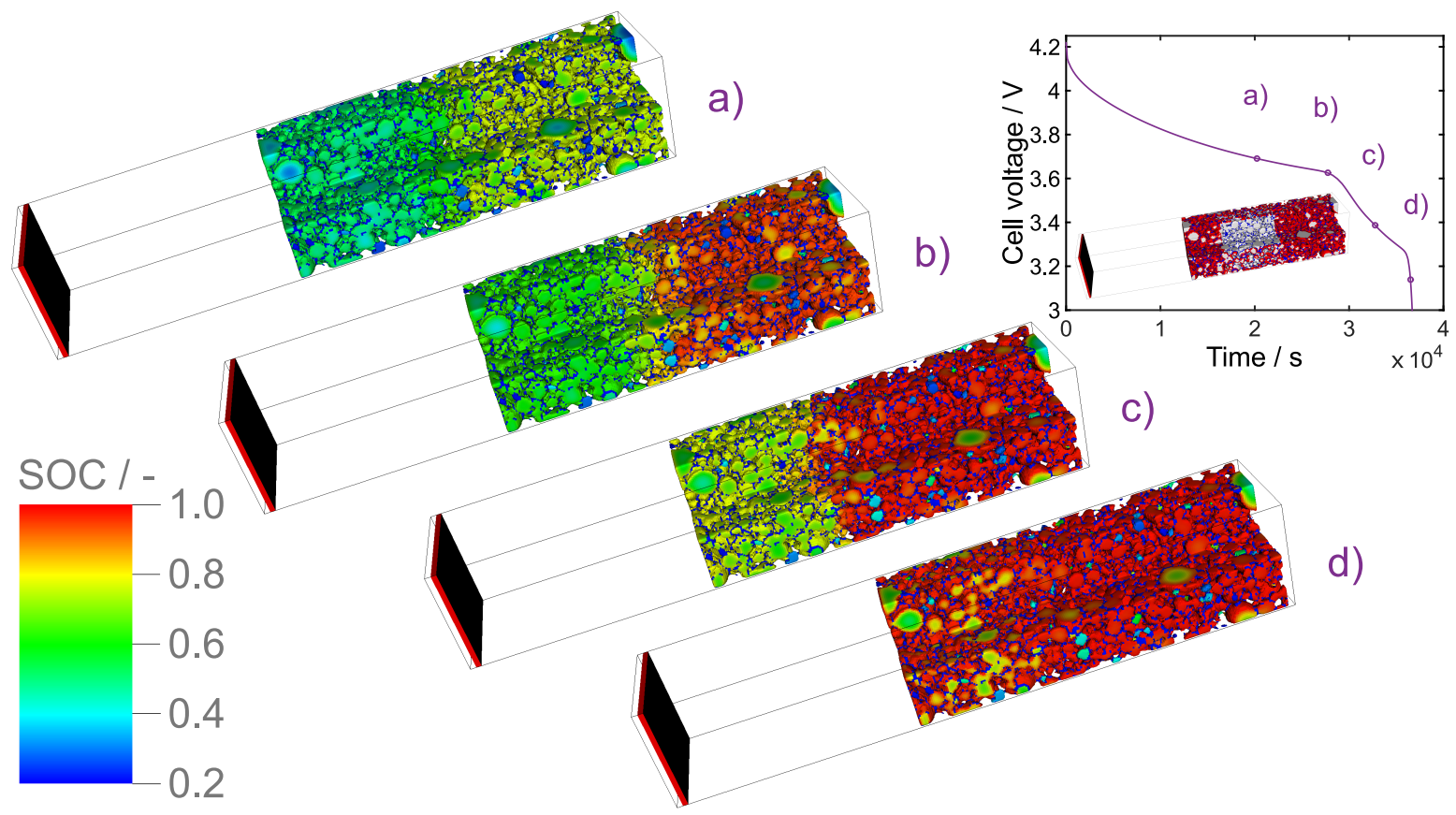

Figure 6: SOC during the lithiation of a thick NMC electrode at a rate of C/10 with a large defect at position 2 (L2) as shown in the inset on the upper right hand corner. The time points a)-d) are given in the corresponding lithiation curve.

inhomogeneities in carbon black distribution in lithiation simulations of thick NMC electrodes. The upper left hand image of Figure 5 shows the resulting current density distribution at a $\mathrm{C} / 10$ rate for an ideal carbon black distribution. The current flows almost exclusively in the surface layer as indicated by the red color. In the inhomogeneous cases the NMC particles in varying parts of the electrode are modeled without a conductive surface layer. As indicated in Figure 5 a)-d) the size is defined by the cross-section of the electrode domain in which the conductive layer of particles is missing. The dimension of the domain in $x$-direction is always 76 voxels and the side length of the cases denoted by 'S', 'M', 'L', and 'full' correspond to 40, 50, 60, and 64 voxels, respectively. As a second parameter we vary the position of the inhomogeneity relative to the current collector. In this case all inhomogeneities do have the same dimension in $x$-direction as position ' 1 ' such that there is some overlap of the regions. The different cases are also illustrated as insets in Figure 5 a)-d). The upper right hand image in Figure 5 shows a representative current density distribution for an electrode with a large inhomogeneity at position 2. The graph demonstrates the change in current density distribution compared to the optimal electrode. As it can be seen at sliced particles some of the current is forced to flow through NMC particles with low electric conductivity. The impact of inhomogeneity position and size on electrode performance is illustrated in the graphs below. Small and medium size defects do not affect the electrode performance even at a high $\mathrm{C} / 2$ rate and irregardless of their position (Figure 5 a) and b)). Only for large defects or even whole layers without conductive additive the effect becomes visible. The portion of the current which has to flow through NMC particles with low conductivity increases with defect size and, therefore, increases the contribution of the ohmic resistance. This effect can already be observed at low C-rates (5)a)) and becomes more pronounced at high C-rates (5b)), eventually leading to a strong decrease in electrode capacity. For 'large' defects we investigate in detail the influence of the position of the inhomogeneities (Figure $5 \mathrm{c})+5 \mathrm{~d})$ ).

In summary we can conclude that a position of the inhomogeneity close to the current collector has a larger influence on the lithiation curve. Moreover, the shape of the lithiation curve shows stages which are normally associated with changes in the host structure of the active material. An effect which is not included in our simulations. This behavior is investigated in Figure 6 which shows the SOC of an electrode with a large defect at position '2' at four characteristic time points during a $\mathrm{C} / 10$ lithiation process. In an electrode with homogeneous carbon black distribution lithiation starts close to the separator and a lithiation front moves towards the current collector during discharge. The presence 


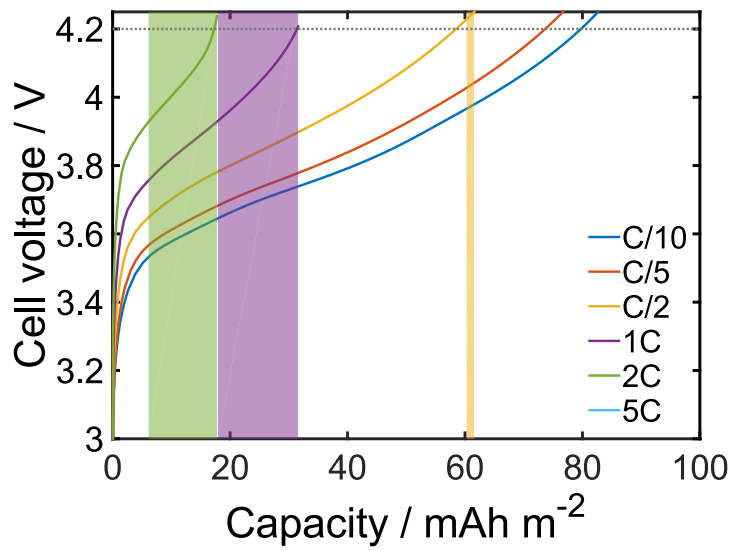

Figure 7: Charge curves of a thick battery cell at increasing C-rates after $\mathrm{C} / 5$ discharge. Colored areas highlight regions during charge, where lithium plating is thermodynamically possible (cf. Eq. 57). The dotted line gives the upper cut-off voltage under operating conditions.

of local inhomogeneities causes a completely different behavior. The region close to the current collector is lithiated first due to its good electrical contact. After complete lithiation of the highly conductive part (Figure 6b)) the reaction front moves towards the top region of the electrode. The current is forced to flow through NMC particles with low conductivity which causes high ohmic losses. This can be observed as a stage in the voltage curve at point c). Finally, at the end of discharge we see an almost complete lithiation of the electrode. At high C-rates the ohmic losses cause a drop in cell voltage below the cut-off voltage before full lithiation can be accomplished. The extreme conditions also affect the stability of the calculations and the simulations stopped prematurely. Nevertheless, we can conclude that areas which are in good electrical contact to the current collector will be de-/lithiated first. Areas with poor electrical contact might not contribute to the cell capacity depending on the magnitude of the applied current. It has to be reminded that we use no-flux boundary conditions in $y$ and $z$ direction. Therefore, the ratio between areas with defects and homogeneous carbon black distribution will not be as dramatic in 'real' electrodes compared to our simulations. Although the volume of the 'medium' sized defect with a dimension of $\sim 106$ x $90 \times 90 \mu \mathrm{m}$ is already quite large, the effect on electrode performance was found to be negligible. This result indicates that the performance of the final electrode is tolerant towards small inhomogeneities. This is good news for the production of thick electrodes as it sets lower requirements regarding a homogeneous carbon black distribution.

\subsection{Lithium plating}

The deposition of metallic lithium at the negative electrode is a well known degradation effect in Li-ion batteries called lithium plating. It causes capacity fading due to a continuous formation of solid electrolyte interphase by both consuming reversibly exchangeable lithium and increasing the resistance for lithium transport through the SEI. In the worst case it is the starting point for dendrite formation which eventually might lead to thermal runaway of the battery and explosion. The necessary thermodynamic condition for lithium plating is a negative overpotential of the Plating reaction $\eta_{\mathrm{pl}}$. It is defined by [17]

$$
\eta_{\mathrm{pl}}=\phi_{\mathrm{Li}}-\varphi_{\mathrm{e}}-U_{\mathrm{Li}}^{0}=\Delta \phi<0,
$$

where $\varphi_{\mathrm{e}}$ is the electrochemical potential of lithium ions in the electrolyte and $U_{\mathrm{Li}}^{0}=0 \mathrm{~V}$ is the OCV of plated lithium against a lithium reference electrode. Due to the high electric conductivity of lithium we assume that the metallic lithium is at the same potential like the graphite electrode $\phi_{\mathrm{Li}}=\phi_{\mathrm{s}}$. In a previous study [10] we demonstrate that the micro-structural effects are able to cause strong local deviations from the average conditions which are calculated in 1+1D simulations. However, exactly these extreme conditions trigger degradation effects. This highlights the need for micro-structure resolved models despite their increased level of complexity and computation time. In our simulations we investigate locally if the condition for lithium plating is met and identify critical operation conditions for thick battery electrodes. Figure 7 shows charge curves of a thick battery after a C/5 discharge. Highlighted areas indicate 


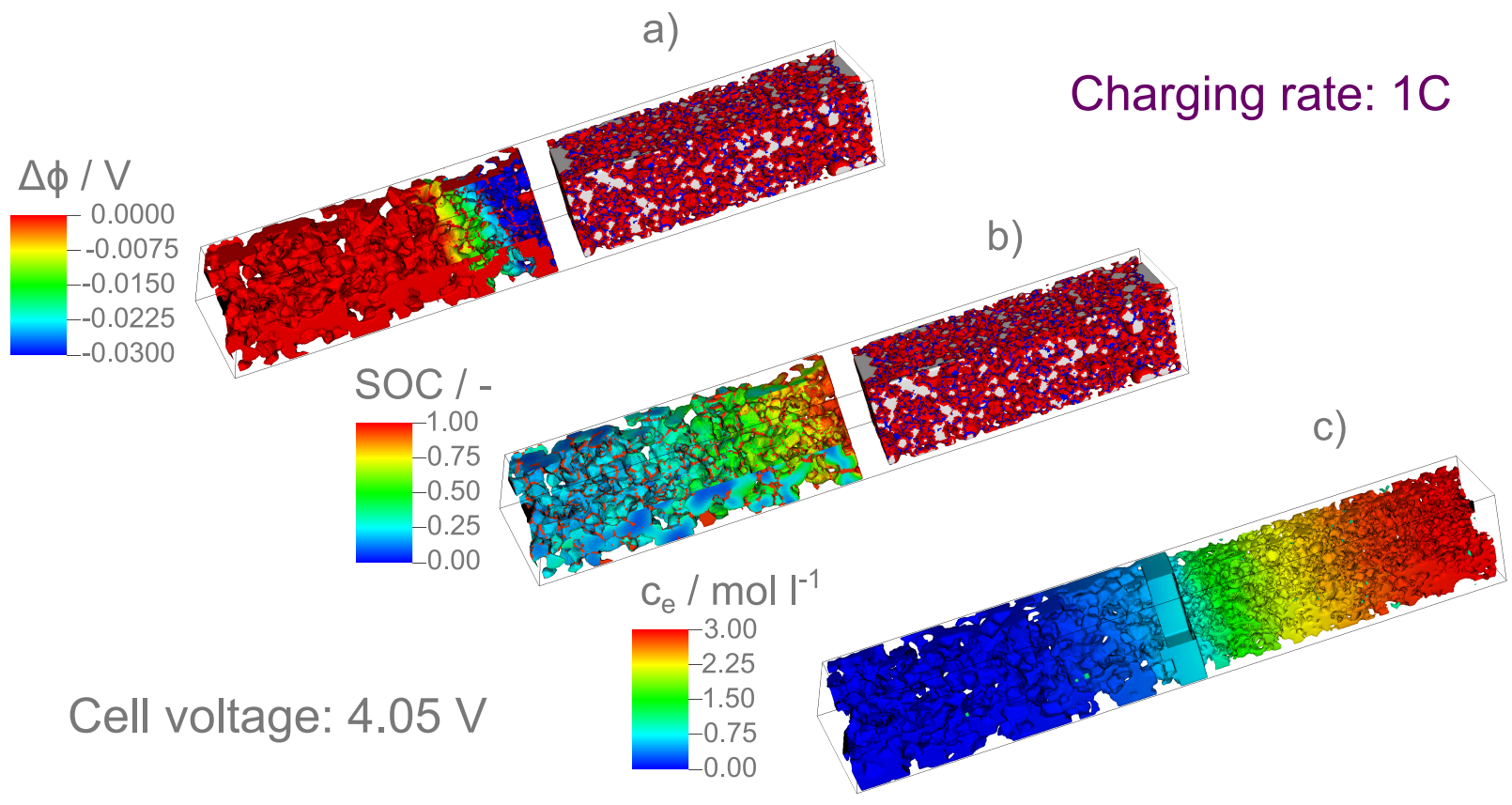

Figure 8: Graphs a)-c) show the distribution of $\Delta \phi$, SOC, and lithium concentration in a thick battery cell at a voltage of $4.05 \mathrm{~V}$ during a $1 \mathrm{C}$ charging simulation.

local conditions where the overpotential of the lithium deposition reaction $\eta_{\mathrm{pl}}$ is negative. Our simulations show that at low charge rates the plating condition is not met. At $\mathrm{C} / 2$ there is a small window towards the end of the charge process, however, the critical region starts above the upper cut-off voltage of $4.2 \mathrm{~V}$. Only at high $\mathrm{C}$-rates considerable parts of the charge process are performed under critical conditions. The distribution of $\Delta \phi$, SOC and lithium ion concentration in the electrolyte during a $1 \mathrm{C}$ charge simulation at a cell voltage of $4.05 \mathrm{~V}$ are shown in Figure 8 . The lithiation of the graphite electrode starts in regions close to the separator. The distribution of SOC (Figure 8 b)) shows that the top region of the electrode is almost completely lithiated while the region near the current collector remains at a SOC close to zero. Note, that the small fiber like bright red features are not lithiated active material but indicate the distribution of the binder phase and unconnected particles. The local plating condition is met in areas which are at least moderately lithiated and negative values of the overpotential are found at the top region of the electrode. During the charge process this region expands towards the current collector. At the end of the charge process almost half of the electrode meets the plating condition. However, a second requirement for lithium plating is the presence of lithium ions. Figure 8 c) shows the distribution of lithium ions in the electrolyte. The graph reveals transport limitations and a very low lithium ion concentration already a few $\mu \mathrm{m}$ behind the anode/separator interface. This limits the occurrence of lithium plating to regions close to the separator even if larger parts of the electrode meet the plating condition of negative overpotential $\eta_{\mathrm{pl}}$ (cf. Eq. (5)).

In the experiments we apply a charging rate of $\mathrm{C} / 10$ in all cases. Therefore, our simulations do not provide any evidence for the occurrence of lithium plating. In order to find a definite answer to the sudden death of the cell after only a few cycles at high discharge rate further studies are needed. From this point of view higher charging rates should be possible without the danger of lithium plating. However, our simulations demonstrate that mass transport limitations will be significant at high currents which reduces the energy efficiency of the cell. The simulations can help to find optimal strategies for battery operation. 


\section{Conclusions}

The use of thick electrodes in Li-ion batteries gives the possibility to reduce the production cost and provides at the same time an improved energy density. However, first experimental studies revealed a short cycle life and a significant decrease in capacity at high C-rates. In this article we present a 3D micro-structure resolved model of a NMC-graphite Li-ion battery implemented in our software BEST. The model was parametrized based on data in the literature and dedicated experiments. We demonstrate the use of supplemental 1+1D simulations for an efficient estimation of kinetic parameters by a fit to de-/lithiation curves measured on thin electrodes $(70 \mu \mathrm{m})$ in half-cell configuration. The results of our model are in good qualitative agreement with the experimental data and give insights on limiting factors during the operation of thick Li-ion batteries. At high C-rates we find a strong limitation of the transport of lithium ions in the electrolyte which is responsible for a significant loss in capacity. Small inhomogeneities in carbon black distribution, however, did not affect the performance of the electrode. Finally, we studied the occurrence of lithium plating during battery charge. At low C-rates we did not find any evidence of lithium plating which leaves the sudden death of the cell as open question for future research. In order to improve the rate capabilities advanced electrode structures, such as layer configurations or gradients in porosity will need to be developed. Micro-structure resolved models in combination with a virtual material design of new electrode concepts will be able to guide future developments in the field.

\section{Acknowledgments}

The authors acknowledge support with computational resources provided by the state of Baden-Württemberg through bwHPC (bwForCluster JUSTUS) and the German Research Foundation (DFG) through grant no INST 40/467-1 FUGG. MS and JK would like to thank the Karlsruhe Institute of Technology and the Helmholtz Institute Ulm for financial support.

[1] M. Singh, J. Kaiser, H. Hahn, Thick Electrodes for High Energy Lithium Ion Batteries, J Electrochem Soc 162 (7) (2015) A1196-A1201. doi:10.1149/2.0401507jes

[2] X. Hu, S. Li, H. Peng, A comparative study of equivalent circuit models for Li-ion batteries, J Power Sources 198 (2012) 359-367. doi: $10.1016 / j$. jpowsour.2011.10.013

[3] J. Remmlinger, S. Tippmann, M. Buchholz, K. Dietmayer, Low-temperature charging of lithium-ion cells Part II: Model reduction and application, J Power Sources 254 (2014) 268-276. doi:10.1016/j.jpowsour.2013.12.101

[4] J. Newman, W. Tiedemann, Porous-electrode theory with battery applications, AIChE J 21 (1) (1975) 25-41. doi:10.1002/aic. 690210103

[5] G. G. Botte, V. R. Subramanian, R. E. White, Mathematical modeling of secondary lithium batteries, Electrochim Acta 45 (15-16) (2000) 2595-2609. doi:10.1016/s0013-4686(00)00340-6

[6] R. E. Garcia, Y.-M. Chiang, W. Craig Carter, P. Limthongkul, C. M. Bishop, Microstructural Modeling and Design of Rechargeable LithiumIon Batteries, J Electrochem Soc 152 (1) (2005) A255. doi:10.1149/1.1836132

[7] G. M. Goldin, A. M. Colclasure, A. H. Wiedemann, R. J. Kee, Three-dimensional particle-resolved models of Li-ion batteries to assist the evaluation of empirical parameters in one-dimensional models, Electrochim Acta 64 (2012) 118-129. doi: 10.1016/j.electacta.2011. 12.119

[8] A. Latz, J. Zausch, Thermodynamic consistent transport theory of Li-ion batteries, J Power Sources 196 (6) (2011) 3296-3302. doi: $10.1016 / j \cdot$ jpowsour. 2010.11 .088

[9] W. Fang, O. Kwon, C. Wang, Electrochemical-thermal modeling of automotive Li-ion batteries and experimental validation using a threeelectrode cell, Int J Energy Res 34 (2010) 107-115. doi:10.1002/er.1652

[10] A. Latz, J. Zausch, Multiscale modeling of lithium ion batteries: thermal aspects, Beilstein J Nanotechnol 6 (2015) 987-1007. doi:10. $3762 /$ bjnano.6.102

[11] C. Monroe, J. Newman, Dendrite Growth in Lithium/Polymer Systems, J Electrochem Soc 150 (10) (2003) A1377. doi:10.1149/1. 1606686

[12] D. R. Ely, A. Jana, R. E. Garcia, Phase field kinetics of lithium electrodeposits, J Power Sources 272 (2014) 581-594. doi:10.1016/j. jpowsour.2014.08.062

[13] H. J. Ploehn, P. Ramadass, R. E. White, Solvent Diffusion Model for Aging of Lithium-Ion Battery Cells, J Electrochem Soc 151 (3) (2004) A456. doi:10.1149/1.1644601

[14] J. Christensen, J. Newman, A Mathematical Model for the Lithium-Ion Negative Electrode Solid Electrolyte Interphase, J Electrochem Soc 151 (11) (2004) A1977. doi:10.1149/1.1804812

[15] M. B. Pinson, M. Z. Bazant, Theory of SEI Formation in Rechargeable Batteries: Capacity Fade, Accelerated Aging and Lifetime Prediction, J Electrochem Soc 160 (2) (2012) A243-A250. doi:10.1149/2.044302jes

[16] P. Arora, M. Doyle, R. E. White, Mathematical Modeling of the Lithium Deposition Overcharge Reaction in Lithium-Ion Batteries Using Carbon-Based Negative Electrodes, J Electrochem Soc 146 (10) (1999) 3543-3553. doi:10.1149/1.1392512

[17] S. Hein, A. Latz, Influence of local lithium metal deposition in 3D microstructures on local and global behavior of Lithium-ion batteries, Electrochim Acta 201 (2016) 354-365. doi:10.1016/j.electacta.2016.01.220

[18] J. Christensen, Modeling Diffusion-Induced Stress in Li-Ion Cells with Porous Electrodes, J Electrochem Soc 157 (3) (2010) A366. doi: $10.1149 / 1.3269995$ 
[19] M. Huttin, M. Kamlah, Phase-field modeling of stress generation in electrode particles of lithium ion batteries, Appl Phys Lett 101 (13). doi:10.1063/1.4754705

[20] K. Takahashi, V. Srinivasan, Examination of Graphite Particle Cracking as a Failure Mode in Lithium-Ion Batteries: A Model-Experimental Study, J Electrochem Soc 162 (4) (2015) A635-A645. doi:10.1149/2.0281504jes

[21] J. Kaiser, V. Wenzel, H. Nirschl, B. Bitsch, N. Willenbacher, M. Baunach, M. Schmitt, S. Jaiser, P. Scharfer, S. Schabel, Prozess- und Produktentwicklung von Elektroden für Li-Ionen-Zellen, Chem-Ing-Tech 86 (5) (2014) 695 - 706. doi:10.1002/cite.201300085

[22] M. Ebner, F. Geldmacher, F. Marone, M. Stampanoni, V. Wood, X-Ray Tomography of Porous, Transition Metal Oxide Based Lithium Ion Battery Electrodes, Adv En Mater 3 (7) (2013) 845-850. doi:10.1002/aenm.201200932

[23] J. Feinauer, T. Brereton, A. Spettl, M. Weber, I. Manke, V. Schmidt, Stochastic 3D modeling of the microstructure of lithium-ion battery anodes via Gaussian random fields on the sphere, Comput Mater Sci 109 (2015) 137-146. doi:10.1016/j.commatsci.2015.06.025

[24] Math2Market GmbH - Becker, J.; Wiegmann, A., GeoDict (2016). URL http: //www.geodict.com

[25] J. S. Newman, K. E. Thomas-Alyea, Electrochemical systems, J. Wiley, 2004.

[26] M. Doyle, T. Fuller, J. Newman, Modeling of galvanostatic charge and discharge of the lithium/polymer/insertion cell, J Electrochem Soc 140 (6) (1993) 1526-1533. doi:10.1149/1.2221597

[27] M. Safari, C. Delacourt, Modeling of a Commercial Graphite/LiFePO4 Cell, J Electrochem Soc 158 (5) (2011) A562. doi:10.1149/1. 3567007

[28] P. De Vidst, R. E. White, Governing Equations for Transport in Porous Electrodes, J Electrochem Soc 144 (4) (1997) 1343-1353. doi: 10.1149/1.1837595

[29] S. G. Meibuhr, Electrode studies in nonaqueous electrolytes, J Electrochem Soc 118 (1) (1970) 1320. doi:10.1149/1.2407438

[30] Fraunhofer Institute for Industrial Mathematics (ITWM) - Zausch, J., BEST - Battery and Electrochemistry Simulation Tool(2016). URL http://www.itwm.fraunhofer.de/en/departments/flow-and-material-simulation/complex-fluids/ best-battery-and-electrochemistry-modeling.html

[31] M. Doyle, Comparison of Modeling Predictions with Experimental Data from Plastic Lithium Ion Cells, J Electrochem Soc 143 (6) (1996) 1890. doi:10.1149/1.1836921

[32] S. Kang, Layered Li(Ni0.5xMn0.5xM2x)O2 ( $\mathrm{M}=\mathrm{Co}, \mathrm{Al}, \mathrm{Ti} ; \mathrm{x}=0,0.025)$ cathode materials for Li-ion rechargeable batteries, J Power Sources 112 (1) (2002) 41-48. doi:10.1016/S0378-7753(02)00360-9

[33] D. Djian, F. Alloin, S. Martinet, H. Lignier, Macroporous poly(vinylidene fluoride) membrane as a separator for lithium-ion batteries with high charge rate capacity, J Power Sources 187 (2) (2009) 575-580. doi:10.1016/j · jpowsour.2008.11.027

[34] J. Vazquez-Arenas, L. E. Gimenez, M. Fowler, T. Han, S.-k. Chen, A rapid estimation and sensitivity analysis of parameters describing the behavior of commercial Li-ion batteries including thermal analysis, Energ Convers Manage 87 (2014) 472-482. doi:10.1016/j. enconman.2014.06.076

[35] D. W. Dees, D. P. Abraham, W. Lu, K. G. Gallagher, M. Bettge, A. N. Jansen, Electrochemical Modeling and Performance of a Lithiumand Manganese-Rich Layered Transition-Metal Oxide Positive Electrode, J Electrochem Soc 162 (4) (2015) A559-A572. doi : 10.1149/2. $0231504 j$ es

[36] L. O. Valoen, J. N. Reimers, Transport Properties of LiPF 6 -Based Li-Ion Battery Electrolytes, J Electrochem Soc 152 (5) (2005) A882. doi:10.1149/1.1872737 


\section{List of Tables}

$1 \quad$ Dimensions and resolution of the electrode samples in $x, y, z$ direction. The reconstructed electrodes are based on cubic voxels with a voxel size of $1.48 \mu \mathrm{m}$. Transport parameters and specific surface areas are determined by simulations in GeoDict [24]. . . . . . . . . . . . . . . . . . . . 16

2 Constitutive equations of 1+1D [26] and 3D [10] Li-ion battery models used in this work. . . . . . . . 16

3 Simulation parameters. Values of electrode and separator thickness, porosity, and cross-sectional area separated by "." indicate parameters for half-cell and full-cell measurements, respectively. In both cases simulations are performed for electrodes with a thickness of 70 and $320 \mu \mathrm{m}$ representing thin and thick electrodes. In order to match the balancing of the electrodes in the full-cell setup the graphite electrode thickness is increased to 85 and $345 \mu \mathrm{m}$, respectively. . . . . . . . . . . . . . . . . . . 17 


\begin{tabular}{l|c|c|c|c|c} 
& $\begin{array}{c}\text { Dimensions } \\
\mu \mathrm{m}\end{array}$ & $\begin{array}{c}\text { Voxels } \\
-\end{array}$ & $\begin{array}{c}\mathrm{a}^{v} \\
\mathrm{~m}^{-1}\end{array}$ & $\begin{array}{c}\kappa_{\text {eff }} \\
\mathrm{S} \mathrm{m}^{-1}\end{array}$ & $\begin{array}{c}\beta \\
-\end{array}$ \\
\hline NMC $(70 \mu \mathrm{m})$ & $\mathrm{x} / \mathrm{y} / \mathrm{z}$ & $\mathrm{x} / \mathrm{y} / \mathrm{z}$ & & & \\
\hline NMC $(320 \mu \mathrm{m})$ & $69.6 / 94.7 / 94.7$ & $47 / 64 / 64$ & 238065 & 1.07 & 2.26 \\
\hline Graphite $(70 \mu \mathrm{m})$ & $69.7 / 94.7 / 94.7$ & $216 / 64 / 64$ & 235370 & 0.99 & 2.23 \\
\hline Graphite $(85 \mu \mathrm{m})$ & $85.8 / 102.1 / 94.7$ & $47 / 64 / 64$ & 116458 & 10.67 & 2.01 \\
\hline Graphite $(320 \mu \mathrm{m})$ & $319.7 / 94.7 / 94.7$ & $58 / 69 / 69$ & 110660 & 9.49 & 1.86 \\
\hline Graphite $(345 \mu \mathrm{m})$ & $344.8 / 102.1 / 102.1$ & $233 / 64 / 64 / 69$ & 105548 & 10.16 & 1.85 \\
\hline \hline
\end{tabular}

Table 1: Dimensions and resolution of the electrode samples in $x, y, z$ direction. The reconstructed electrodes are based on cubic voxels with a voxel size of $1.48 \mu \mathrm{m}$. Transport parameters and specific surface areas are determined by simulations in GeoDict [24].

\section{$1+1 \mathrm{D}$}

$\frac{\partial \varepsilon_{\mathrm{e}} c_{\mathrm{e}}}{\partial t}=-\frac{\partial}{\partial x}\left(-D_{\mathrm{e}} \varepsilon_{\mathrm{e}}^{\beta} \frac{\partial c_{\mathrm{e}}}{\partial x}+\frac{t_{\mathrm{Li}^{+}} i_{\mathrm{e}}}{F}\right)+\frac{a^{v} i_{s e}}{F}$

Material balance

$$
0=-\frac{\partial i_{\mathrm{e}}}{\partial x}+a^{v} i_{s e}
$$$$
i_{\mathrm{e}}=-\kappa \varepsilon_{\mathrm{e}}^{\beta} \frac{\partial \phi_{\mathrm{e}}}{\partial x}-\kappa_{D} \varepsilon_{\mathrm{e}}^{\beta} \frac{\partial c_{\mathrm{e}}}{\partial x}
$$$$
\kappa_{D}=\frac{2 \kappa R T\left(t_{\mathrm{Li}^{+}}-1\right)}{F c_{\mathrm{e}}}\left(1+\frac{\partial \ln f_{\mathrm{Li}^{+}}}{\partial \ln c_{\mathrm{e}}}\right)
$$

\section{Active material}

$$
\begin{gathered}
\frac{\partial c_{\mathrm{s}}}{\partial t}=-\frac{1}{r^{2}} \frac{\partial}{\partial r}\left(-r^{2} D_{\mathrm{s}} \frac{\partial c_{\mathrm{s}}}{\partial r}\right) \\
0=-\frac{\partial i_{\mathrm{s}}}{\partial x}-a^{v} i_{s e} \\
i_{\mathrm{s}}=-\sigma^{\mathrm{eff}} \frac{\partial \phi_{\mathrm{s}}}{\partial x}
\end{gathered}
$$

\section{BEST (3D)}

$$
\frac{\partial c_{\mathrm{e}}}{\partial t}=-\nabla \cdot\left(-D_{\mathrm{e}} \nabla c_{\mathrm{e}}+\frac{t_{\mathrm{Li}^{+}} i_{\mathrm{e}}}{F}\right)
$$

$$
i_{\mathrm{e}}=-\kappa \nabla \phi_{\mathrm{e}}-\kappa_{D} \nabla c_{\mathrm{e}}
$$$$
\kappa_{D}=\frac{\kappa\left(t_{\mathrm{Li}^{+}}-1\right)}{F}\left(\frac{\partial \mu_{\mathrm{e}}}{\partial c_{\mathrm{e}}}\right)
$$

$$
\begin{gathered}
\frac{\partial c_{\mathrm{s}}}{\partial t}=-\nabla \cdot\left(-D_{\mathrm{s}} \nabla c_{\mathrm{s}}\right) \\
0=-\nabla \cdot i_{\mathrm{s}} \\
i_{\mathrm{s}}=-\sigma \nabla \phi_{\mathrm{s}}
\end{gathered}
$$

Table 2: Constitutive equations of 1+1D [26] and 3D [10] Li-ion battery models used in this work. 


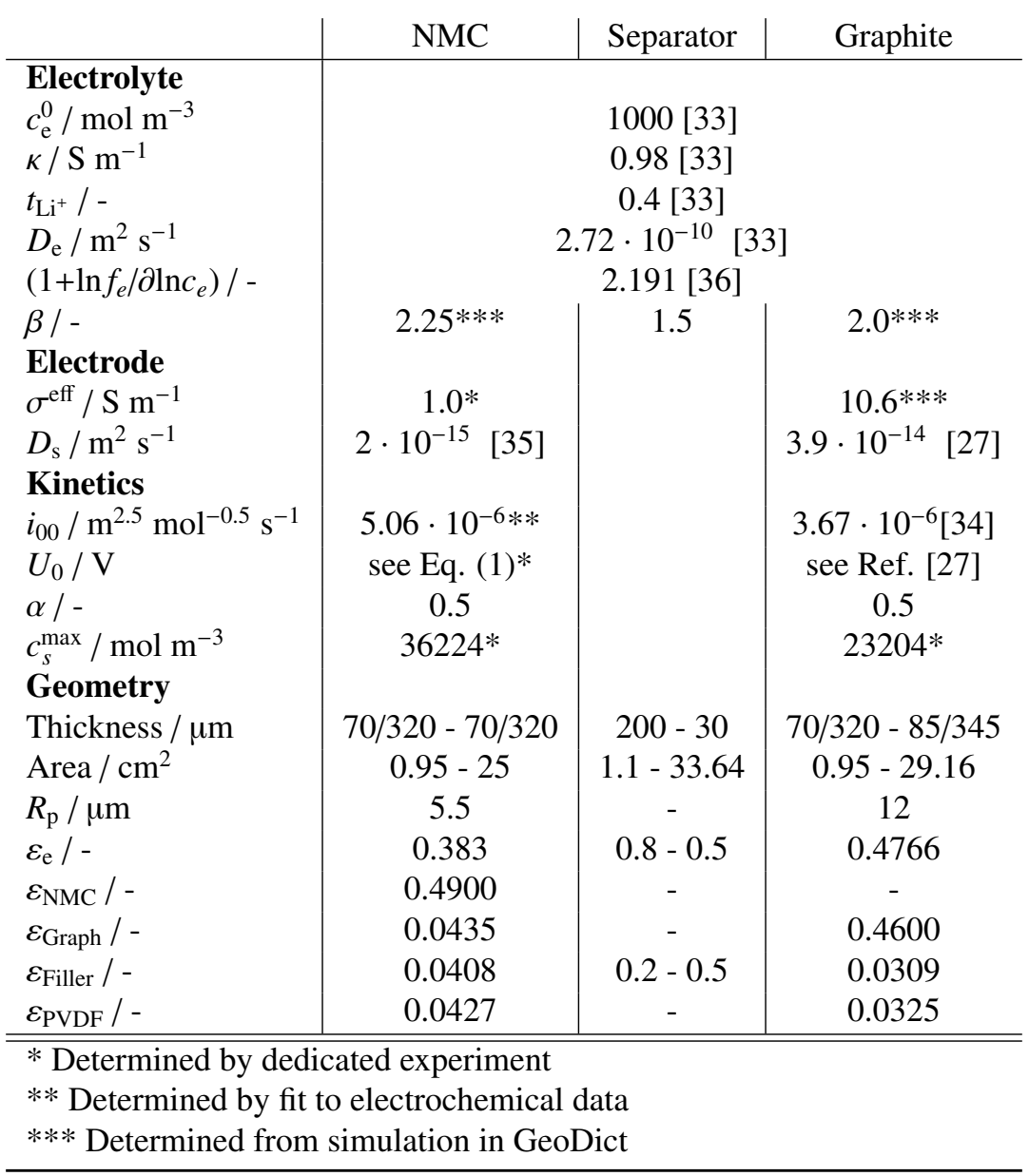

Table 3: Simulation parameters. Values of electrode and separator thickness, porosity, and cross-sectional area separated by "." indicate parameters for half-cell and full-cell measurements, respectively. In both cases simulations are performed for electrodes with a thickness of 70 and $320 \mu \mathrm{m}$ representing thin and thick electrodes. In order to match the balancing of the electrodes in the full-cell setup the graphite electrode thickness is increased to 85 and $345 \mu \mathrm{m}$, respectively. 


\begin{tabular}{|cll|}
\hline Symbol & Unit & Meaning \\
$a^{v}$ & $\mathrm{~m}^{2} \cdot \mathrm{m}^{-3}$ & Specific surface area \\
$c_{\mathrm{e}}$ & $\mathrm{mol} \cdot \mathrm{m}^{-3}$ & $\mathrm{Li}^{+}$concentration in electrolyte \\
$c_{\mathrm{s}}$ & $\mathrm{mol} \cdot \mathrm{m}^{-3}$ & $\mathrm{Lithium}^{-3}$ concentration in active material \\
$D_{\mathrm{e}}$ & $\mathrm{m}^{2} \cdot \mathrm{s}^{-1}$ & $\mathrm{Li}^{+}$diffusion coefficient \\
$D_{\mathrm{s}}$ & $\mathrm{m}^{2} \cdot \mathrm{s}^{-1}$ & Lithium diffusion coefficient \\
$f_{\mathrm{e}}$ & - & Activity coefficient \\
$F$ & $\mathrm{C} \cdot \mathrm{mol}^{-1}$ & Faradays constant \\
$i_{k}$ & $\mathrm{~A} \cdot \mathrm{m}^{-2}$ & Current density in phase $k$ \\
$i_{s e}$ & $\mathrm{~A} \cdot \mathrm{m}^{-2}$ & Faradaic current density \\
$i_{0}$ & $\mathrm{~A} \cdot \mathrm{m}^{-2}$ & Exchange current density \\
$i_{00}$ & $\mathrm{~m}{ }^{2.5} \mathrm{~mol}^{-0.5} \mathrm{~s}^{-1}$ & Rate constant \\
$r$ & $\mathrm{~m}$ & Radial coordinate \\
$R$ & $\mathrm{~J} \cdot \mathrm{mol}^{-1} \cdot \mathrm{K}^{-1}$ & Ideal gas constant \\
$R_{\mathrm{p}}$ & $\mathrm{m}$ & Particle radius \\
$t$ & $\mathrm{~s}$ & Time \\
$t_{\mathrm{Li}}{ }^{+}$ & - & Transference number \\
$U_{0}$ & $\mathrm{~V}$ & Open circuit voltage \\
$x, y, z$ & $\mathrm{~m}$ & Spatial coordinates \\
$\alpha$ & - & Symmetry factor \\
$\beta$ & - & Bruggeman coefficient \\
$\varepsilon_{k}$ & - & Volume fraction of phase $k$ \\
$\eta$ & $\mathrm{V}$ & Overpotential \\
$\kappa$ & $\mathrm{S} \cdot \mathrm{m}^{-1}$ & Electrolyte conductivity \\
$\mu_{\mathrm{e}}$ & $\mathrm{J} \cdot \mathrm{mol}^{-1}$ & Chemical potential \\
$\sigma_{k}$ & $\mathrm{~S} \cdot \mathrm{m}^{-1}$ & Electric conductivity of phase $k$ \\
$\phi_{\mathrm{s}}$ & $\mathrm{V}$ & Electrolyte potential \\
$\phi_{\mathrm{e}}$ & $\mathrm{V}$ & Electrode potential \\
$\varphi_{\mathrm{e}}$ & $\mathrm{J} \cdot \mathrm{mol}^{-1}$ & Electrochemical potential \\
\hline & & \\
\hline
\end{tabular}

